\title{
The effect of different probiotic strains on immune function in vitro
}

\author{
Honglin Dong, Ian Rowland, Kieran M. Tuohy and Parveen Yaqoob \\ Department of Food and Nutritional Sciences, The University of Reading, Whiteknights, PO Box 226, \\ Reading RG6 6AP, UK
}

Lactobacilli and bifidobacteria are two major probiotic species used in the food industry. The modulation of immune function by probiotics has become a hot topic in the recent years. The immune response to probiotics is generally regarded to be strain-dependent. However, evidence for this is rather limited, particular with regard to the cellular immune response. The aim of the current study was to compare the immonomodulatory properties of different probiotic strains in a human blood mononuclear cell model in vitro.

Live cells of four Lactobacillus strains (Lactobacillus casei Shirota, Lactobacillus rhamnosus GG, Lactobacillus plantarum NCIMB 8826, Lactobacillus reuteri NCIMB 11951/ATCC23272) and two Bifidobacterium strains (Bifidobacterium longum SP 07/3, and Bifidobacterium bifidum MF 20/5) were individually incubated with human peripheral blood mononuclear cells from seven healthy older subjects at a ratio of 1:1 for $24 \mathrm{~h}$. Expression of the activation markers, CD69 and CD25, and natural killer cell activity were assessed by flow cytometry. Supernatants were collected for cytokine measurement (flow cytometry or ELISA).

All six strains stimulated CD69 expression on $\mathrm{CD} 3{ }^{+} \mathrm{CD}^{+}{ }^{+}$(cytotoxic T cells; Tc) and $\mathrm{CD} 3{ }^{-} \mathrm{CD} 6^{+}$(natural killer; NK cells) subsets, but increased CD25 expression only on NK cells. All strains also increased the level of expression (mean fluoresecence intensity; MFI) of CD69 on $\mathrm{CD}^{+}$(Th) and $\mathrm{CD}^{+} \mathrm{CD}^{+} 6^{+}$(NKT cells) subsets, and all strains, except for L. casei Shirota and L. reuteri NCIMB 11951, induced CD25 expression (MFI) by NKT cells. NK cell activity was significantly increased by all six strains, without any difference between strains. All of the strains increased the production of IL-1 $\beta$, IL-6, IL-10, TNF- $\alpha$, GM-CSF, MIP- $1 \alpha$ to different extents, but had no effect on IL-2, IL-4, IL-5, or TNF- $\beta$ production. Four of the cytokines/chemokines analysed were differentially regulated by the probiotics; these were interferon- $\gamma$ (IFN- $\gamma$ ), regulated upon activation, normal T-cell expressed and secreted (RANTES), IL-12 and MCP1. Table 1 summarises the effects of the six strains on these mediators. IFN- $\gamma$ and RANTES production was stimulated by all strains except for L. rhamnosus GG and LPS $(P=0.062-0.05)$. IL-12 production was only increased by $L$. casei Shirota and $L$. plantarum NCIMB $8826(P<0.001)$. IL-8 production was induced by all lactobacillus strains except for $L$. reuteri NCIMB $11951(P=0.083-0.05)$. MCP-1 production was only induced by bifidobacteria and LPS $(P=0.075-0.05)$. In addition, bifidobacteria and LPS were more potent inducers of IL-10 than lactobacilli. All strains, except for $L$. reuteri NCIMB 11951, induced more IL- $1 \beta$ and TNF- $\alpha$ production than LPS.

The results suggested that there was little evidence of strain-specific effects on $\mathrm{T}$ cell activation or natural killer cell activity, while the production of some cytokines and chemokines was differentially influenced by probiotic strains. The biological importance of these selected strain-specific effects remains to be determined.

\begin{tabular}{lccccccc}
\hline & \multicolumn{7}{c}{ Probiotic strains } \\
\cline { 2 - 8 } $\begin{array}{l}\text { Cytokine } \\
\text { production }\end{array}$ & LPS & $\begin{array}{c}\text { L. casei } \\
\text { Shirota }\end{array}$ & $\begin{array}{c}\text { L. rhamnosus } \\
\text { GG }\end{array}$ & $\begin{array}{c}\text { L. plantarum } \\
\text { NCIMB 8826 }\end{array}$ & $\begin{array}{c}\text { L. reuteri } \\
\text { NCIMB 11951 }\end{array}$ & $\begin{array}{c}\text { B. longum } \\
\text { SP 07/3 }\end{array}$ & $\begin{array}{c}\text { B. bifidum } \\
\text { MF 20/5 }\end{array}$ \\
\hline IFN- $\gamma$ & - & $\uparrow$ & - & $\uparrow$ & $\uparrow$ & $\uparrow$ & $\uparrow$ \\
RANTES & - & $\uparrow$ & - & $\uparrow$ & $\uparrow$ & - & $\uparrow$ \\
IL-12 & - & $\uparrow$ & - & $\uparrow$ & - & - & - \\
IL-8 & - & $\uparrow$ & $\uparrow$ & - & - & - & - \\
MCP-1 & $\uparrow$ & - & - & - & & $\uparrow$ & - \\
\hline
\end{tabular}

$\uparrow$ increase, - no change compared with the negative control.

This research was sponsored by a Dorothy Hodgkin Postgraduate Award and Yakult UK. 